\author{
Yu-Xin CAI, PhD (Corresponding author) \\ E-mail: yx_cai@shu.edu.cn \\ School of Management, Shanghai University \\ Yun-Lu GONG, PhD \\ E-mail: ylgong@shu.edu.cn \\ School of Science, Shanghai University \\ Guo-Yang SHENG \\ E-mail: 845339732@qq.com \\ Antai School of Economics and Management \\ Shanghai Jiaotong University
}

\title{
THE GOLD PRICE AND THE ECONOMIC POLICY UNCERTAINTY DYNAMICS RELATIONSHIP: THE CONTINUOUS WAVELET ANALYSIS
}

\begin{abstract}
This article uses the continuous wavelet analysis to investigate lead-lag relationship between gold future markets and economic policy uncertainty in different time-frequency domains by covering a period of 1998M01-2020M02. Our findings show the positive relationship between gold future returns and economic policy uncertainty before the period of global financial crisis, while after the crisis, this relationship changed to irregular correlated in the US and UK. Furthermore, we find that gold future returns are leading economic policy uncertainty changes in the very short and short terms in US, and in the short term in UK before 2008, while after 2008, the lead-lag relationships change to ambiguous correlated, indicating the gold could be employed as a safe-haven asset hedge against uncertainty before 2008, but its role changed after 2008. Due to the COVID-19 pandemic in the world, increasingly uncertainty may change its role again, and gold's price are likely to peak in the future.
\end{abstract}

Keywords: Gold futures, Economic policy uncertainty, Lead-lag relationship, Continuous wavelet analysis.

JEL Classification: F37

\section{Introduction}

Gold's role as an inflation hedge, safe-haven asset has attracted attention worldwide for a long time. Several studies investigate whether the gold price can be viewed as a leading indicator of financial security or stability (Dyhrberg, 2016; Wu et al., 2019; Beckmann et al., 2019; Roh et al., 2020). On the other hand, some

DOI: 10.24818/18423264/55.1.21.07 
studies argue that as the increasing uncertainty of global finance, economic policy uncertainty variables could predict financial products (Balcilar et al.,2016; Bouri et al., 2017; Bams et al., 2017; Cai et al., 2020), furthermore, predict the gold market (Fang et al., 2018; Bouoiyour et al., 2018; Gozgor et al., 2019). However, no conclusive results have been reached about the form and the causal direction of the volatility linkage between them. So, it is important to explore the dynamic relationship between the gold market and economic policy uncertainty.

Dyhrber (2016) exam whether gold, bitcoin or the dollar is the most useful hedging tool, the result show that bitcoin can combine some of the advantages of both gold and dollar in the financial markets, and so be a useful tool for risk and market sentiment analysis. Bouoiyour et al.(2018) find a positive and strong relationship between gold return and the uncertainty variables when the uncertainty attains its highest level and under normal gold market scenario, suggesting that a diversified portfolio composed of gold could help protecting against exposure to uncertain risks. But Beckmann et al.(2019) find gold is a hedge or safe haven in the classical sense before 2008 financial crisis, while after 2008, its role changed significantly. Though most studies examine the linkages between gold market and other financial series, few study uses economic policy uncertainty index to examine the co-movement and causality between gold return and economic policy uncertainty. As we divide frequency dimension into five frequency bands: very short term, short term, medium term, long term, and very long term, we can test whether gold market can be viewed as a leading indicator hedge against the economic policy uncertainty across different times and frequencies.

Different with Dyhrberg (2016), Fang et al. (2018) and Wu et al.(2019) used GARCH models to test whether gold is a safe haven hedge against risk or uncertainty. We use the continuous wavelet analysis to extend the time-frequency test between gold future returns and economic policy uncertainty. The continuous wavelet analysis can describe how the volatility linkage between two series develops over time and varies across different frequency bands in a highly intuitive way. It provides information about the lead-lag relationship between two series. Therefore, this method has been widely used to identify the leading indicator between two interactive factors or estimate the co-movement and causality between two series (Aloui et al., 2016; Chen et al., 2017; Zaremba et al., 2019; Wang and $\mathrm{Li}, 2020)$. And it does not require the two series to be stationary of cointegrated which exhibits a major advantage of widely accommodating financial series, regardless of stationary properties.

US economic policy uncertainty (USEPU) which explored by Baker et al. (2016) is a weighted average of three components. The first component quantifies the volume of news discussing policy-related uncertainty per month since January 1985. The second component measures the level of uncertainty related to future changes in the tax code. This is done by using data from the Congressional Budget Office on the tax provisions which set to expire in the near future. The third component captures forecasters' divergences about future monetary and fiscal 
The Gold Price and the Economic Policy Uncertainty Dynamics Relationship: The Continuous Wavelet Analysis

policies. The authors use the Survey of Professional Forecasters provided by the Federal Reserve Board of Philadelphia to obtain forecasts of CPI, as well as purchases of goods and services by federal, state, and local governments. And UK economic policy uncertainty (UKEPU) quantifies by the similar manner. Several literatures ensured that economic policy uncertainty (EPU) did in fact capture aggregate economic policy uncertainty (Gulen and Ion, 2016; Nguyen and Phan, 2017; Jens, 2017; Junttila and Vataja, 2018; Jin et al., 2019).

This paper focuses on the co-movements between gold future market and economic policy uncertainty. For the gold price, we choose gold market from New York Commodity Exchange (COMEX) and London Bullion Market Association (LBMA) which are the most active gold futures markets with the largest trading volume in the world. And we use USEPU, UKEPU to quantify aggregate economic policy uncertainty in US, UK, respectively. Our study contributes to understand the co-movement between gold markets returns and economic policy uncertainty using both qualitative and quantitative analyses. Through the continuous wavelet analysis, we found that gold future returns and economic policy uncertainty are positively correlated in very short and short terms in US, in the short term in UK before global financial crisis, while they are positively or negatively correlated in different frequency bands during and after global financial crisis. There are breakdowns of the link between the gold market and economic policy uncertainty. Before global financial crisis, gold as a hedge or safe haven can hedge against economic policy uncertainty, during and after global financial crisis, gold may not be able to play that role. The gold cannot be used as a leading indicator of the safehaven asset to hedge against uncertainty or risk. But the increasing uncertainty caused by COVID-19 may change gold's role again, so that gold's price may reach unprecedented height in the future.

\section{Methodology}

The continuous wavelet analysis mainly involves wavelet coherency and phase-difference. Based on a mother wavelet $\varphi$, a family $\varphi_{\tau, s}$ of "wavelet daughter" is defined as:

$$
\varphi_{\tau, s}(t):=\frac{1}{\sqrt{|s|}} \varphi\left(\frac{t-\tau}{s}\right) . \tau, s \in \mathbb{R}, s \neq 0
$$

where $\tau$ is a translation parameter, and $s$ is a scaling factor. We choose Morlet wavelet as the mother wavelet, which is given as:

$$
\varphi(t)=\pi^{-\frac{1}{4}} \exp \left(i \bar{\omega}_{0} t\right) \exp \left(-\frac{1}{2} t^{2}\right)
$$

where $\int_{-\infty}^{+\infty}|\varphi(t)|^{2} d t<+\infty$, and $\int_{-\infty}^{+\infty}|\varphi(t)| d t=0$.

Based on the mother wavelet, the continuous wavelet transform is defined as:

$$
W_{x ; \varphi}(\tau, s)=\int_{-\infty}^{+\infty} x(t) \frac{1}{\sqrt{|s|}} \varphi^{*}\left(\frac{t-\tau}{s}\right) d t
$$

DOI: 10.24818/18423264/55.1.21.07 
where $*$ denotes the complex conjugation of the Morlet wavelet. The time series is expanded into a time-frequency space depending on $\tau$ and $s$. Then, the wavelet power spectrum is given as:

$$
W P S_{x}(\tau, s)=\left|W_{x}(\tau, s)\right|^{2}
$$

Further, the cross-wavelet transform of two different time series, $x(t)$ and $y(t)$ is defined as:

$$
W_{x y}(\tau, s)=W_{x}(\tau, s) W_{y}^{*}(\tau, s)
$$

where $W_{x}(\tau, s)$ and $W_{y}(\tau, s)$ are the continuous wavelet transforms of $x(t)$ and $y(t)$. And the dynamic correlation in different time-frequency domain can be investigated by the wavelet coherency. The wavelet coherency is defined as follows:

$$
R_{x y}=\frac{\left|S\left(W_{x y}\right)\right|}{\sqrt{S\left(\left|W_{x}\right|^{2}\right) S\left(\left|W_{y}\right|^{2}\right)}}
$$

where $s$ is a scaling factor. And the phase-difference is applied to reveal the positive or negative correlation and lead-lag interaction between two different financial time series. The angle $\phi_{x y}$ of the complex coherency is the phasedifference, which is defined as:

$$
\phi_{x y}=\tan ^{-1}\left(\frac{\Im\left(S\left(W_{x y}\right)\right)}{\Re\left(S\left(W_{x y}\right)\right)}\right)
$$

where $\mathfrak{I}()$ and $\mathfrak{R}()$ are the imaginary part and real part, respectively. And different arrows (range of angles) indicate different lead-lag relationship between two series.

\section{Data}

We use monthly data on the gold future return and economic policy uncertainty variables covering January 1998 to February 2020 with 265 observations. For the gold return, we choose gold future market from COMEX and LBMA which are the most active gold futures market in the world. For economic policy uncertainty variables, we choose USEPU and UKEPU followed by Baker et al. (2016). We restrict the sample to the 1998M01-2020M02 period as the UKEPU is available since January 1998. The original data were derived from the Economic Policy Uncertainty website and Wind Data Services. The monthly returns of gold $R_{t}$ are defined as the logarithmic difference of the monthly closing price $p_{t}$.

$$
G R_{t}=\log \left(p_{t}\right)-\log \left(p_{t-1}\right)
$$

We set monthly changes in $E P U$ as follows:

$$
\triangle E P U_{t}=E P U_{t}-E P U_{t-1}
$$

where $E P U$ denotes USEPU or UKEPU, respectively. $G R_{t}, \triangle E P U_{t}$ observations are illustrated in Table 1.

Table 1 shows the descriptive statistics for monthly gold return (COMEX, LBMA), monthly USEPU, UKEPU changes ( $\triangle$ USEPU, $\triangle$ UKEPU). Each gold return of the mean value is close to zero, and its standard deviation is nearly zero. Each skewness is nearly zero and kurtosis are all smaller than 15, indicating no strong deviations from normality. The ADF test shows the stationarity of monthly gold return and monthly EPU changes at the $1 \%$ significance level. 
The Gold Price and the Economic Policy Uncertainty Dynamics Relationship: The Continuous Wavelet Analysis

Table 1. Descriptive statistic for monthly returns of gold and monthly USEPU, UKEPU changes

\begin{tabular}{|l|l|l|l|l|l|l|c|}
\hline Variable & Mean $(\%)$ & Max & Min & S.D. & Skewness & Kurtosis & ADF \\
\hline COMEX & .2705 & .0660 & -.0886 & .0208 & -.0750 & 4.4377 & $-18.27^{*}$ \\
\hline LBMA & .2716 & .0676 & -.0799 & .0203 & -.0355 & 3.9288 & $-17.84^{*}$ \\
\hline$\Delta$ USEPU & 36.98 & 177 & -160 & 38.88 & .4512 & 7.1388 & $-20.45^{*}$ \\
\hline$\Delta$ UKEPU & 10.57 & 257 & -247 & 45.55 & -.0691 & 10.0938 & $-19.19^{*}$ \\
\hline
\end{tabular}

Note: * Denotes the rejection of the null hypothesis at the $1 \%$ significance level.

\section{Empirical results}

\subsection{The wavelet power spectrum analysis}

We separate the vertical axis into 5 frequency bands: $0-0.5$ years, $0.5-1$ years, 1-2 years, 2-4 years, and 4-8 years, corresponding to the volatilities in the very short term, short term, medium term, long term, and very long term. The color represents the strength of power, ranging from blue (low power) to yellow (high power). The cone of influence (COI) is given by a white line, which defines the regions affected by the edge effects. We mainly focus on the regions inside the COI. Figure 1 displays the wavelet power spectrum of COMEX, LBMA, USEPU and UKEPU.

As shown in Figure 1 (a.2-d.2), the horizontal axis denotes the time component while the vertical axis denotes the frequency bands, from period half year (6 months) up to period 8 years (96 months). Figure 1 (a.1) (b.1) show the gold future price seems to present a stable pattern. We focus on the variation as shown in Figure 1 (a.2) (b.2), the volatilities of gold future markets are significant (at 5\% significance level) in the 0-0.5 frequency band during 2008-2009 and 20112012. This implies the significant volatilities of gold price during the period of the global financial crisis and European sovereign debt crisis in the very short term. The European sovereign crisis is an ongoing the global financial crisis which made some countries in the euro area difficult or impossible to re-finance their government debt without the assistance of third parties. Due to the stimulus economic policies and gold's role as a safe-haven asset, a relatively high level of gold volatilities during that time in the very short term.

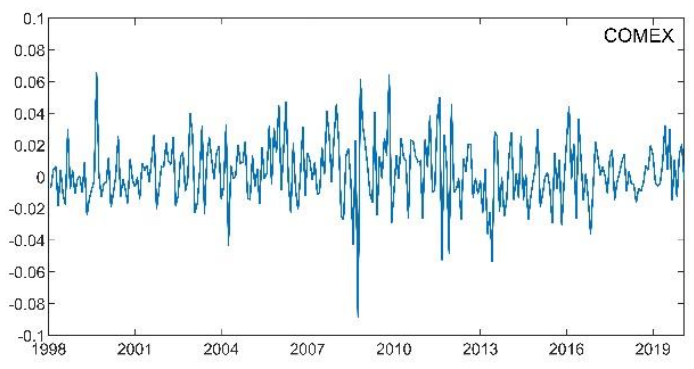

(a.1) 
Yu-Xin Cai, Yun-Lu Gong, Guo-Yang Sheng

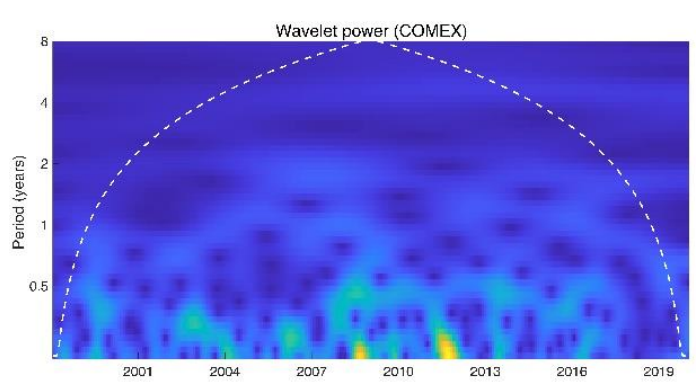

(a.2)

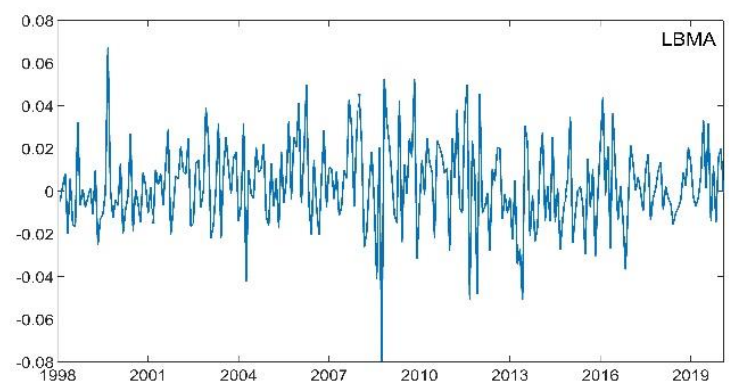

(b.1)

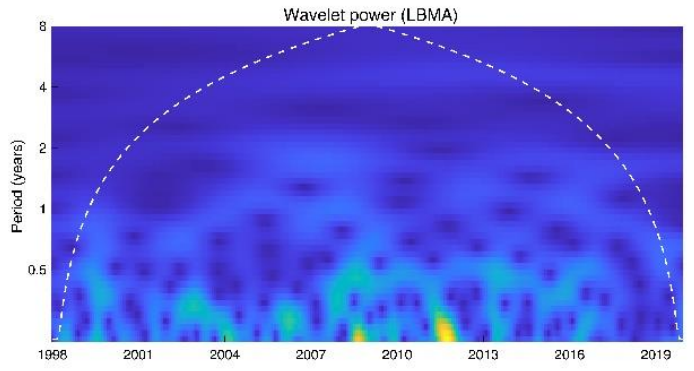

(b.2)

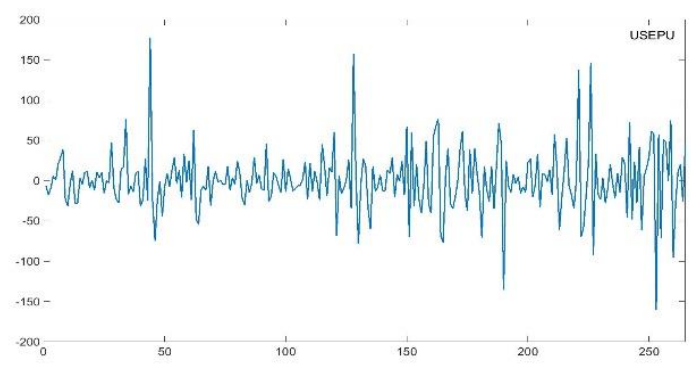

(c.1) 
The Gold Price and the Economic Policy Uncertainty Dynamics Relationship: The Continuous Wavelet Analysis

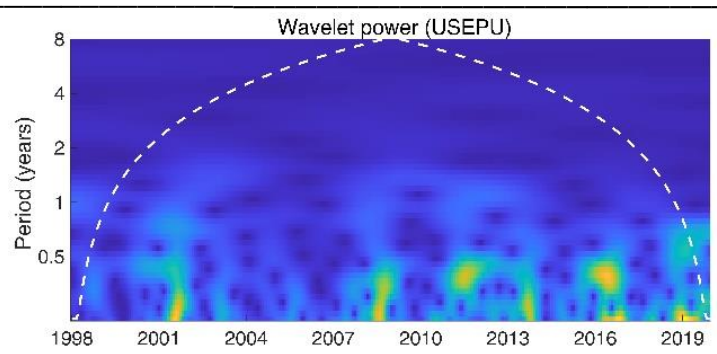

(c.2)

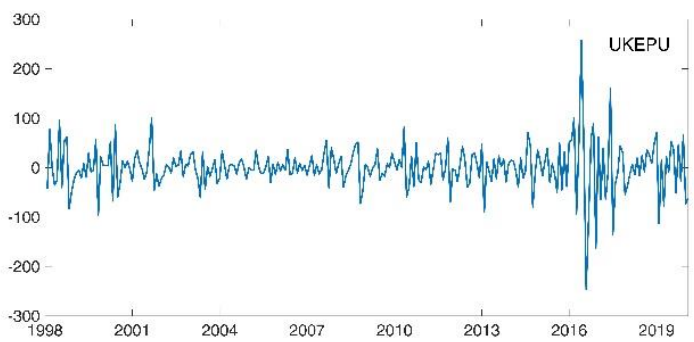

(d.1)

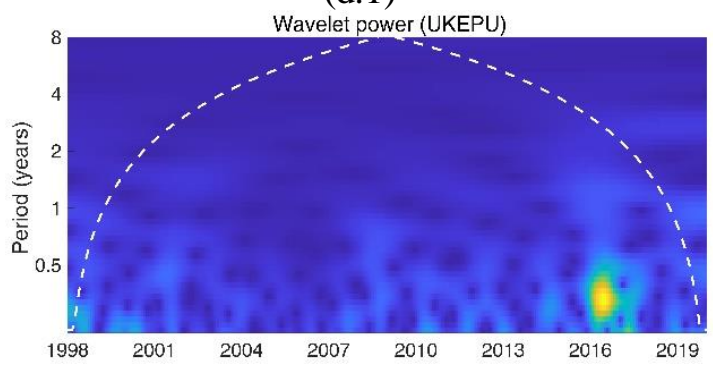

(d.2)

Figure 1. (a.1-d.1) The volatility of gold (COMEX, LBMA), and economic policy uncertainty (USEPU, UKEPU), respectively, (a.2-d.2) The wavelet power spectrum of COMEX, LBMA, USEPU, and UKEPU, respectively. The white line refers to the COI. The color code of power varies from blue (low power) to yellow (high power)

Figure 1 (c.2) shows significant (at 5\% significance level) volatilities of USEPU changes in the 0-0.5 frequency band over 2001-2002, 2008-2009, 20112014, 2016-2017 and 2019-2020. This means the high level of USEPU changes during that times in the very short term. Since the fourth quarter of 2000, the growth rate of the US economy has declined sharply. Before 9.11 (2001), the US economy was on the verge of recession. The 9.11 incident further had a very serious negative impact on the US economy and increased the economic uncertainty. The significant economic policy uncertainty during global financial crisis and European sovereign debt crisis periods indicate the deep and lasting

DOI: 10.24818/18423264/55.1.21.07 
impact of the crisis on the US economy. And the increased uncertainty caused by the COVID-19 is just beginning.

The UKEPU changes seems to present a stable pattern except 2016-2017 as shown in Figure 1 (d.2). The volatilities of UKEPU are significant (at 5\% significance level) in the 0-0.5 frequency band during 2016-2017. This implies the significant volatilities of The UKEPU during the period of 2016-2017 in the very short term. The UKEPU changes was relatively high over 2016-2017 caused by the UK voted to leave the European Union Member States since June 2016. In the UK, the increased economic policy uncertainty brought by Britain's secession from European Union is far higher than the risk of financial crisis which can be recovered by economic stimulus policies.

\subsection{The wavelet coherency and the phase-difference}

We identify causality and phase differences between gold returns and EPU changes. Arrows indicate the phase differences between gold futures returns and EPU changes. For example, $\rightarrow$ and $\longleftarrow$ indicate both gold returns and EPU changes are in phase and out of phase, respectively. $\nearrow$ and $\measuredangle$ indicate gold returns are leading economic policy uncertainty changes, while $\searrow$ and $\nwarrow$ indicate gold returns are lagging EPU changes.

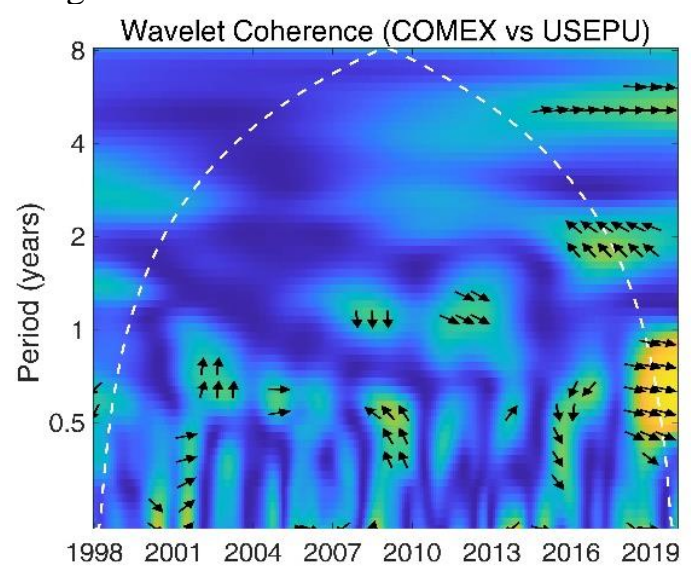

(a) 
The Gold Price and the Economic Policy Uncertainty Dynamics Relationship: The Continuous Wavelet Analysis

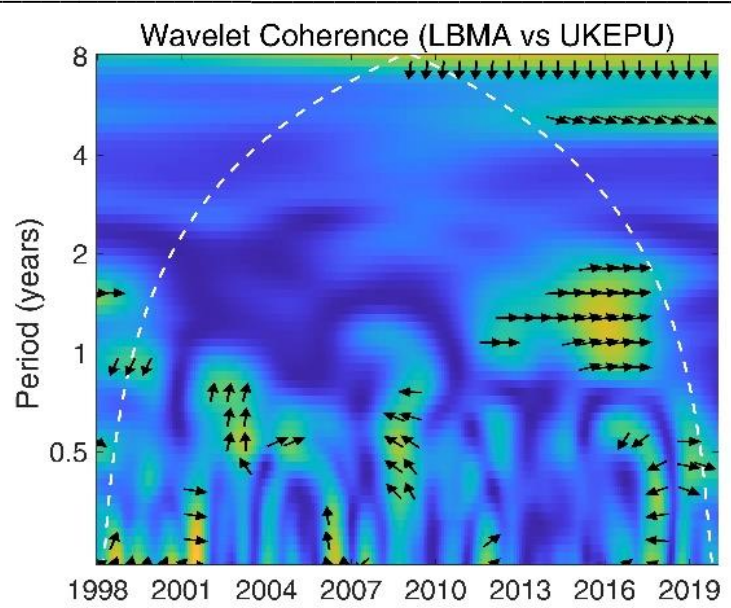

(b)

Figure 2. Wavelet coherency of COMEX-USEPU and LBMA-UKEPU, respectively. The arrows contour denotes the 5\% significance level. The color code of power varies from blue (low power) to yellow (high power).

As shown in Figure 2 (a), we observe the significant degree of comovement between COMEX gold returns and USEPU changes for 2000-2001, 2008-2010 and 2015-2016 period across the 0-0.5 year frequency band, for 20012004, and 2019-2020 period across the 0.5-1 year frequency band, for 2010-2012, and 2016-2017 period across the 1-2 years frequency band. We find the arrow point $\lambda$ for 2000-2001 across the 0-0.5 year frequency band, and for 2001-2004 across the 0.5-1 year frequency band, indicating gold returns and USEPU changes are positively correlated, gold returns are leading USEPU changes in the very short term and short term around 9.11 incident. The arrow point $\nwarrow$ for 2008-2010 across the 0-0.5 year frequency band, and $\searrow$ for 2010-2012 across the 1-2 years frequency band, indicating gold returns and USEPU changes are negatively correlated, gold returns are lagging USEPU changes in the very short term after 2 years of global financial crisis, while changed to positively correlated in the medium term the next two years. $\searrow$ for $2015-2016$ across the $0-0.5$ years frequency band, and $\nwarrow$ for 2016 2017 across the 1-2 years frequency band, indicating gold returns and EPU changes are positively correlated, gold returns are lagging EPU changes in the very short term during the period of US economic recovery, while changed to negatively correlated in the medium term the next two years. Before the global financial crisis, gold returns are leading EPU changes, gold as a safe-haven asset can hedge against uncertainty. During and after the global financial crisis, gold future price and EPU interact through multiple ways and the positive or negative correlations vary in different frequency bands, gold's role cannot as a safe haven hedge against uncertainty after 2008. Since 2020, the COVID-19 is beginning in US, $\rightarrow$

DOI: $10.24818 / 18423264 / 55.1 .21 .07$ 
indicates that gold returns and USEPU changes are in phase during that time in the short term, the highest level of uncertainty may change gold's role again, so that gold's price may reach unprecedented height in the future.

In Figure 2 (b), the significant degree of co-movement between LBMA gold future returns and UKEPU is 2000-2001, 2006-2007 and 2017-2019 period across the 0-0.5 year frequency band, for 2001-2004, and 2008-2009 period across the 0.5-1 year frequency band, for 2012-2016 period across the 1-2 years frequency band. $\searrow$ for 2000-2001 across the 0-0.5 year frequency band shows that there is a positive relationship between LBMA gold return and UKEPU in the very short term. $\nearrow$ for 2001-2004 across the 0-1 year frequency band also indicates LBMA and UKEPU changes are positively correlated, and gold returns are leading EPU changes in the very short and short terms. $\$ for 2006-2007 across the 0-0.5 year frequency band, and for 2008-2009 across the 0.5-1 year frequency band indicates LBMA and UKEPU changes are negatively correlated, gold returns are lagging EPU changes in the very short and short terms around global financial crisis. While after 2008, the relationship between LBMA and EPU changed to positive correlated in the medium term which the arrow point shows $\rightarrow$ for 2012-2016 period across the 1-2 years frequency band. And we can see $\measuredangle$ for 2017-2018, \ for 2018-2019 across the 0.5-1 year frequency band, indicating LBMA return and EPU changes interact through multiple ways and the irregular correlations vary for different times. Before the global financial crisis, there is a positive relationship between LBMA gold future return and EPU, while during and after the global financial crisis, the irregular and disordered relationship between LBMA and EKEPU is discovered. This result is similar to the US, which also indicates gold as a safe-haven asset can hedge against uncertainty before 2008, but its role changed after 2008. Due to the COVID-19 pandemic in UK after the time in US, LBMA gold returns and EPU are in phase during that time in the very short term, and less obviously than the US.

\section{Conclusions}

Previous studies discuss that whether the gold can play a role in hedging in capital market. or be a useful tool to hedge against uncertainty and risk. In this paper, the co-movement between gold future markets and EPU are investigated using the continuous wavelet analysis which can provides more information on the dynamic relationship. The main findings of the study are as follows. Firstly, the empirical results show that the gold future markets in US and UK all experienced significant volatilities around the period of global financial crisis, while the USEPU experienced significant volatilities after 9.11 incident and around the period of global financial crisis, and the UKEPU experienced significant volatilities just in the period of 2016-2017. Secondly, gold returns are positively correlated with EPU changes before the period of global financial crisis, while after the period of global financial crisis, this relationship changed to negatively or positively correlated because of the increasingly irregular and disordered capital market in the US, UK. Finally, before the period of global financial crisis, gold 
The Gold Price and the Economic Policy Uncertainty Dynamics Relationship: The Continuous Wavelet Analysis

returns are leading EPU changes in the very short and short terms in the US, and in the short term in the UK, while after the period of crisis, the lead-lag relationships between gold future returns and EPU change to ambiguous correlated. And the lead-lag relationships between them may change again because of COVID-19 pandemic. In general, the gold future market could be employed as a safe-haven asset to hedge against uncertainty before 2008, but its role changed after 2008. Due to the COVID-19 pandemic in the world, its role may change again, and gold's price are likely to peak in the future.

This paper provides empirical evidence from the perspective of dynamic correlation and lead-lag relationship using the continuous wavelet analysis. The above results provide several implications to policy makers and investors. In times of heightened EPU, policy makers should sustain the momentum of capital performances, regulate financial market to maintain financial stability. Because of the linkage effect of various financial markets, policy makers can provide investment incentives to boost investors' participations in the financial market, establish a stability capital market fund by benchmarking it to a certain safety level, etc. These are all useful to avoid financial markets' systemic risks.

\section{REFERENCES}

[1]Aloui, C., Hkiri, B., Nguyen, D.K. (2016), Real Growth Co-movement and Business Cycle Synchronization in the GCC Countries: Evidence from Timefrequency Analysis. Economic Modelling, 52, 322-331;

[2]Baker, S., Bloom, N., Davis, S. (2016), Measuring Economic Policy

Uncertainty. Quarterly Journal of Economics, 131(4), 1593-1636.

[3]Beckmann, J., Berger, T., , Czudaj, R. (2019), Gold Price Dynamics and the

Role of Uncertainty. Quantitative Finance, 19, 663-681;

[4]Bouri, E., Roubaud, D., Jammazi, R., Assaf, A. (2017), Uncovering

Frequency Domain Causality between Gold and the Stock Markets of China and

India: Evidence from Implied Volatility Indices. Finance Research Letters, 23,

23-30;

[5]Balcilar, M., Gupta, R., Pierdzioch, C. (2016), Does Uncertainty Move the Gold Price? New Evidence from a Nonparametric Causality-in-quantiles Test. Resources Policy, 49, 74-80;

[6]Bouoiyour, J., Selmi, R., Wohar, M. E., (2018), Measuring the Response of Gold Prices to Uncertainty: An Analysis Beyond the Mean. Economic Modelling, 75, 105-116;

[7]Bams, D., Blanchard, G., Honarvar, I., Lehnert, T. (2017), Does Oil and Gold Price Uncertainty Matter for the Stock Market? Journal of Empirical Finance, 44, 270-285;

DOI: $10.24818 / 18423264 / 55.1 .21 .07$ 
Yu-Xin Cai, Yun-Lu Gong, Guo-Yang Sheng

[8]Chen, M.P., Chen, W.Y., Tseng, T.C. (2017), Co-movements of Returns in the Health Care Sectors from the US, UK, and Germany Stock Markets:

Evidence from the Continuous Wavelet Analyses. International Review of Economics \& Finance, 49,484-498;

[9]Cai, Y.,X., Tao, Y.,Y., Yan Z.,X. (2020), Stock Market Trading Volumes and Economic Policy Uncertainty Dependence: Before and During Sino-US Trade Friction. Economic Research-Ekonomska Istraživanja, 33(1), 1711-1728; [10]Dyhrberg, A.H. (2016), Bitcoin, Gold and the Dollar: A GARCH Volatility Analysis. Finance Research Letters, 16, 85-92. doi:10.1016/j.frl.2015.10.008; [11]Drobetz W., Ghoul S. E., Guedhami O., Janzen M. (2018), Policy Uncertainty, Investment, and the Cost of Capital. Journal of Financial Stability, 39,28-45;

[12]Fang, L., Chen, B., Yu, H., Qian, Y. (2018), The Importance of Global Economic Policy Uncertainty in Predicting Gold Futures Market Volatility: A GARCH-MIDAS Approach. Journal of Futures Markets, 38, 413-422;

[13]Gozgor, G., Lau, C. K. M., Sheng, X., Yarovaya, L. (2019), The Role of Uncertainty Measures on the Returns of Gold. Economics Letters, 185, 108680; [14]Gulen H., Ion M. (2016), Policy Uncertainty and Corporate Investment. Review of Financial Studies, 29(3), 523-564;

[15]Jin J.X., Chen Z.Q., Yang X.L.(2019), Economic Policy Uncertainty and Stock Price Crash Risk. Accounting and Finance, 58,1291-1318;

[16]Jens, C. E. (2017), Political Uncertainty and Investment: Causal Evidence from US Gubernatorial Elections. Journal of Financial Economics, 124(3),563579 ;

[17]Nguyen, N. H., Phan, H. V. (2017), Policy Uncertainty and Mergers and Acquisitions. Journal of Financial and Quantitative Analysis, 52(2), 613-644; [18]Roh, T., Byun, S. J., Xu, Y. (2020), Downside Uncertainty Shocks in the Oil and Gold Markets. International Review of Economics \& Finance, 66, 291-307; [19]Wang, R., Li, L.,F. (2020), Dynamic Relationship between the Stock Market and Macroeconomy in China (1995-2018):Mew Evidence from the Continuous Wavelet Analysis. Economic Research-Ekonomska Istraživanja, 33(1),521-539; [20]Wu, S., Tong, M., Yang, Z., Derbali, A. (2019), Does Gold or Bitcoin Hedge Economic Policy Uncertainty? Finance Research Letters, 31, 171-178;

[21]Zaremba, A., Umar, Z., Mikutowski, M. (2019), Inflation Hedging with Commodities: A Wavelet Analysis of Seven Centuries Worth of Data. Economics Letters, 181,90-94. 